\title{
Cell motility as random motion: A review
}

\section{Cell motility as random motion}

\author{
D. Selmeczi ${ }^{1,2}$, L. Li $^{3}$, L.I.I. Pedersen ${ }^{4,7}$, S.F. Nrrelykke ${ }^{5}$, P.H. Hagedorn ${ }^{7}$, S. Mosler ${ }^{1}$, \\ N.B. Larsen ${ }^{1,7}$, E.C. Cox ${ }^{6}$, and H. Flyvbjerg ${ }^{1,7, a}$ \\ 1 Danish Polymer Centre, Ris $\varnothing$ National Laboratory, Technical University of Denmark, \\ 4000 Roskilde, Denmark \\ 2 Department of Biological Physics, Etvs Lornd University (ELTE), 1117 Budapest, Hungary \\ 3 Department of Physics, Princeton University, Princeton, NJ 08544, USA \\ 4 The Niels Bohr Institute, Blegdamsvej 17, 2100 Copenhagen $\varnothing$, Denmark \\ 5 Max-Planck-Institute for the Physics of Complex Systems, Nthnitzer Strasse 38, \\ 01187 Dresden, Germany \\ 6 Department of Molecular Biology, Princeton University, Princeton, NJ 08544, USA \\ 7 Biosystems Department, Ris $\varnothing$ National Laboratory, Technical University of Denmark, \\ 4000 Roskilde, Denmark
}

\begin{abstract}
The historical co-evolution of biological motility models with models of Brownian motion is outlined. Recent results for how to derive cell-type-specific motility models from experimental cell trajectories are reviewed. Experimental work in progress, which tests the generality of this phenomenological model building is reported. So is theoretical work in progress, which explains the characteristic time scales and correlations of phenomenological models in terms of the dynamics of cytoskeleton, lamellipodia, and pseudopodia.
\end{abstract}

\section{The co-evolution of theories for Brownian and biological random motion}

Robert Brown did not discover Brownian motion. ${ }^{1}$ Brown, a botanist, got his name associated with this physical phenomenon because he in 1827 carefully demonstrated what it is not, a manifestation of life. The puzzle of its true origin he left for others to solve. It being a property of inert matter, those others were the physicists. They ignored the problem for decades, then slowly began to show it some interest [1].

Brownian motion had been known for as long as the microscope [2,3], and before the kinetic theory of heat it was natural to assume that "since it moves, it is alive." Brown initially thought so too, before he killed that notion. The cause of the misunderstanding, the similarity between the motion of microscopic life forms and microscopic particles, is deep, however, as subsequent developments demonstrate.

After Einstein had published his theory for Brownian motion in 1905 and 1906 [4], Przibram in 1913 demonstrated that this theory describes also the self-propelled random motion of protozoa [5]. By tracking the trajectories $\mathbf{x}(t)$ of individual protozoa, see Fig. 1, Przibram

\footnotetext{
a e-mail: henrik.flyvbjerg@risoe.dk

1 The opening sentence on Brownian motion in The Feynman Lectures on Physics (Benjamin Cummings, 2005) states that he did. This is a rare mistake by Feynman, and his introduction to the subject is good, nevertheless.
} 


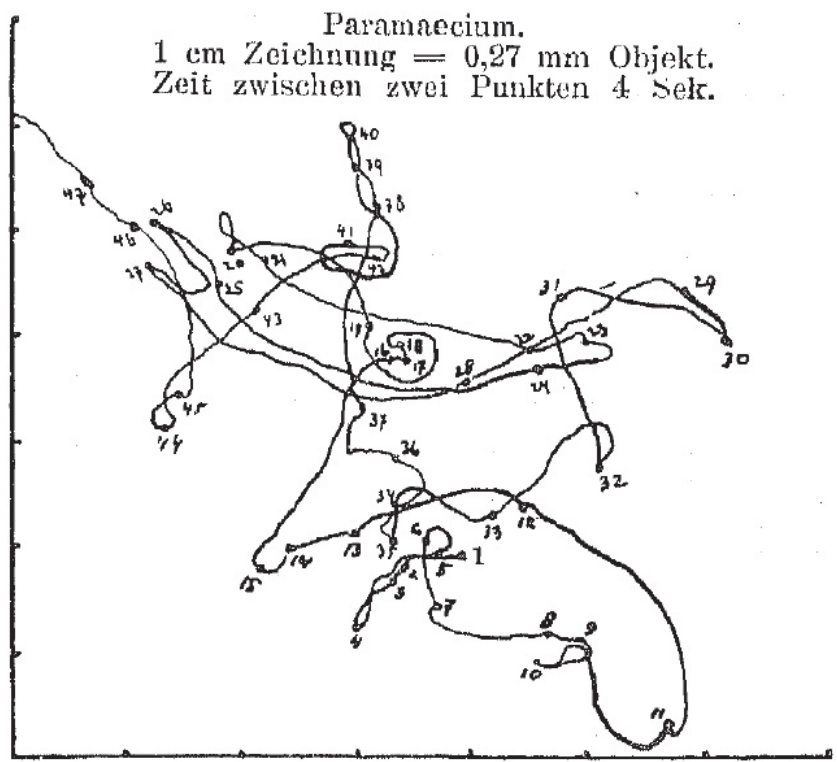

Fig. 1. Example of Przibram's motility data, a trajectory of a protozoon, hand-drawn with a mechanical tracking device operated in real time with a microscope. A metronome was used to mark time on the trajectory every four seconds [5].

demonstrated that the net displacement $\mathbf{x}(t)-\mathbf{x}(0)$ averages to zero, while its square satisfies the relationship known for Brownian motion,

$$
\left\langle\mathbf{d}(t)^{2}\right\rangle=2 n_{\operatorname{dim}} D t
$$

where $n_{\text {dim }}$ is the dimension of the space in which the motion takes place.

In Einstein's theory $D$ is the diffusion coefficient, and satisfies his famous relation $D=$ $k_{\mathrm{B}} T / \gamma_{0}$, where $k_{\mathrm{B}}$ is Boltzmann's constant, $T$ the absolute temperature, and $\gamma_{0}=6 \pi \eta a$ is Stokes drag coefficient for a rigid sphere with radius $a$ moving with constant velocity through a fluid at rest and having dynamic viscosity $\eta$. Przibram found a value for $D$ which was much larger and much more sensitive to changes in temperature than Einstein's relation states. He used this as proof that it was not just Brownian motion that he had observed.

If Przibram, a biologist, had used a better time resolution by marking out points in Fig. 1 more frequently than every four seconds, he might have gotten ahead of the physicists in theoretical developments. But he was drawing by hand, marking time to a metronome, so marking points closer to $1 \mathrm{~Hz}$ must have been a challenge.

Fürth, a physicist at the German university in Prague where Einstein had been a professor for 16 months in 1911-12, also studied the motility of protozoa. First he repeated Przibram's results, apparently without knowing about them [6]. Later he found that his data were not described by Eq. (1) [7]. He consequently considered a random walker on a lattice, and gave the walker directional persistence in the form of a bias towards stepping in the direction of the step taken previously. By taking the continuum limit, he, independently of Ornstein $[8,9]$, demonstrated that for random motion with persistence, Eq. (1) is replaced by

$$
\left\langle\mathbf{d}(t)^{2}\right\rangle=2 n_{\operatorname{dim}} D\left(t-P\left(1-e^{-t / P}\right)\right),
$$

where $P$ is called the persistence time, and characterizes the time for which a given velocity is "remembered" by the system [7].

Ornstein solved the Langevin equation [10],

$$
m \dot{\mathbf{x}}(t)=-\gamma_{0} \dot{\mathbf{x}}(t)+\mathbf{F}_{\text {thermal }}(t)
$$


in answer to a question raised by Smoluchowski about the role of inertia in Brownian motion. In Eq. (3) $m$ is the inertial mass of the Brownian particle. The force from the surrounding medium is written as a sum of two terms: Stokes friction, $-\gamma_{0} \dot{\mathbf{x}}$, and a random thermal force $\boldsymbol{F}_{\text {thermal }}=\left(2 k_{\mathrm{B}} T \gamma_{0}\right)^{1 / 2} \eta(t)$ with Gaussian white-noise statistical properties,

$$
\langle\eta(t)\rangle=\mathbf{0} ; \quad\left\langle\eta_{j}\left(t^{\prime}\right) \eta_{k}\left(t^{\prime \prime}\right)\right\rangle=\delta_{j, k} \delta\left(t^{\prime}-t^{\prime \prime}\right) .
$$

Here $\delta(t)$ and $\delta_{j, k}$ are, respectively, Dirac's and Kronecker's $\delta$-functions, and the vector $\eta(t)$ is assumed uncorrelated with $\mathbf{x}\left(t^{\prime}\right)$ for $t \geq t^{\prime}$. Fürth's formula, Eq. (2), is a consequence of Eq. (3), which conveniently may be rewritten as

$$
P \frac{d \mathbf{v}}{d t}=-\mathbf{v}+(2 D)^{1 / 2} \eta,
$$

with $P \simeq m / \gamma_{0}$, but Eq. (2) follows also from other, similar, theories.

Note that the physical meaning of the three terms in Eq. (3) does not apply to cell motion: Firstly, cell velocities are measured in micrometers per hour, so their inertial mass means absolutely nothing for their motion. Secondly, friction with the surrounding medium is also irrelevant for cells that move about firmly attached to the substrate they move on. Thirdly, it is not thermal forces that accelerate the cells. But as a mathematical model of cell motility, Eq. (5) is about the simplest possible of its kind. The persistent random motion that it describes, is called the Ornstein-Uhlenbeck process (OU-process), and is, because of its mathematical simplicity, a conceptual paradigm like the harmonic oscillator, the Hydrogen atom, and the Ising model. It also agrees with the earliest data, though Eq. (2) often was the only aspect of the theory that was compared with experimental data, and with good reason, considering the limited quality of the data. Thus the OU-process became the standard model for motility, or, more correctly, Eq. (2) became the standard formula of cell motility.

Gail and Boone [11] seem to have been the first to interpret cell motility data with Eq. (2). They did a time study of fibroblasts from mice by measuring the cells' positions every $2.5 \mathrm{hrs}$. Equation (2) fitted their results fairly well. Since then, cell motility data have routinely been fitted with Eq. (2). Its agreement with data can be impressive, and is usually satisfactorysometimes helped by the size of experimental error bars and few points at times $t$ that are comparable to $P$. Data with these properties cannot distinguish Eq. (2) from other functions that quickly approach $2 n_{\operatorname{dim}} D(t-P)$.

Equation (2) is essentially a double integral of the velocity auto-correlation function $\phi(t)$ of the OU-process, where

$$
\phi(t)=\langle\mathbf{v}(0) \cdot \mathbf{v}(t)\rangle=\frac{n_{\operatorname{dim}} D}{P} e^{-|t| / P} .
$$

Experimental results for the velocity auto-correlation function are better suited for showing whether the OU-process is a reasonable model for given data. But experimental results for velocities are calculated as finite differences from time-lapse recordings of positions. If the time-lapse is short, precision is low on differences, hence on computed velocities. Yet, if the time-lapse is longer, the time resolution of the motion is poor. The solution is somewhere in between, compensating for lost precision with good statistics. Good statistics was not really achievable till computer-aided object-tracking became possible.

\section{Enter computers}

We recently wanted to characterize the compatibility of human cells with various surfaces by describing the cells' motility on the various surfaces [12]. Computer-aided cell tracking-see Fig. 2-quickly gave us so much data that we found ourselves in a new situation with regards to modeling: We were not limited to showing whether or not there is agreement between data and a few consequences of a given model. We could investigate the model itself experimentally, measure each term in its defining equation, check that their assumed properties are satisfied, and whether together they satisfy the equation of motion. 

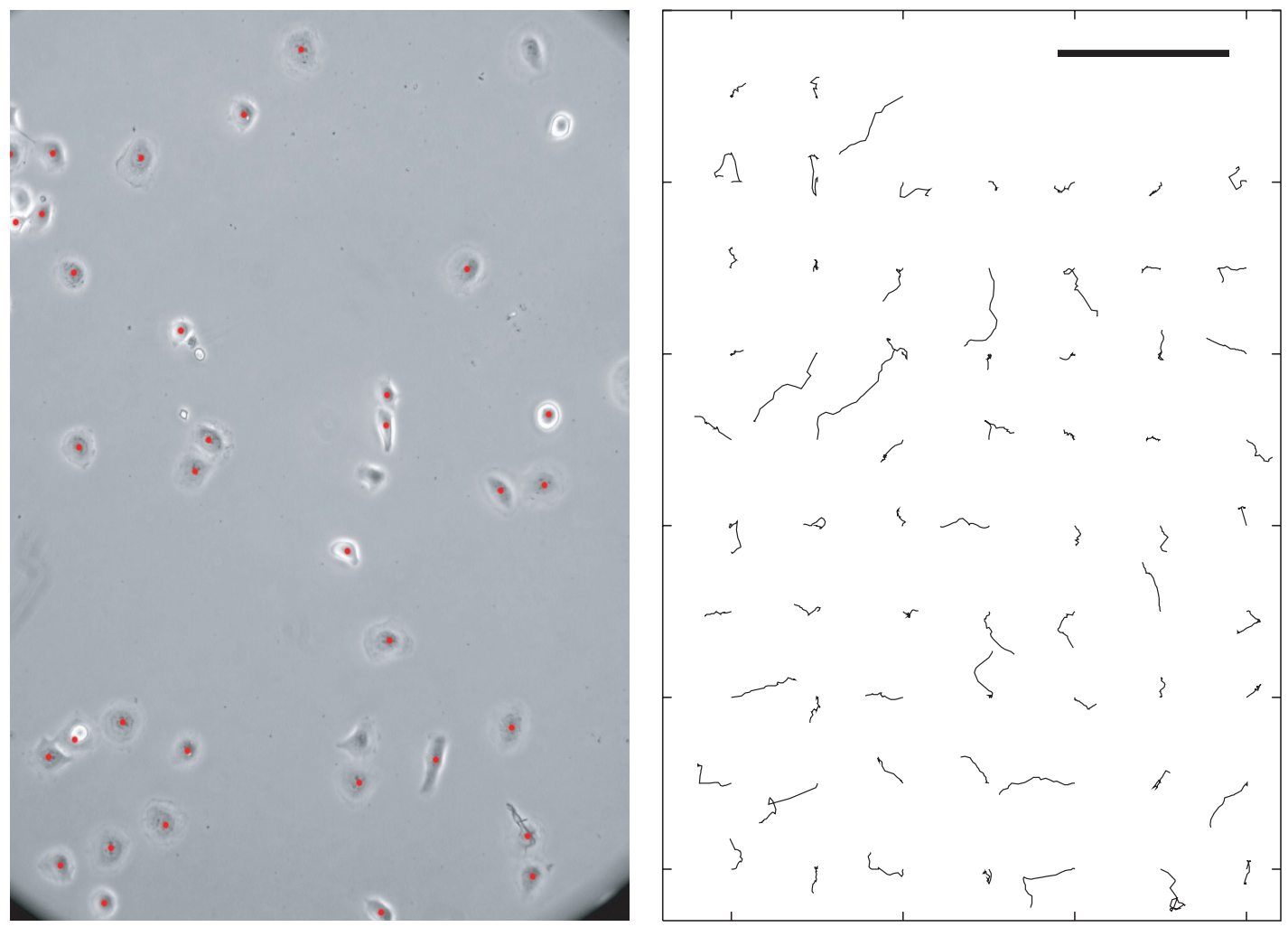

Fig. 2. Isolated human dermal keratinocytes are motile by nature. If not surrounded by other cells, they react as if in a wound: They search for other cells of the same kind with which they can connect to form skin. Trajectories are formed from 15 min time-lapse photography. Left: photograph of cells with the center of each cell's nucleus marked with a circular dot. The positional error of this mark is much smaller than its radius. The error was found by repeatedly marking the same cell and calculating the RMSD of the resulting set of positions. The diameter of the round cells in the photograph is approximately $30 \mu \mathrm{m}$. The cells are shown with higher density than was used in the experiments that produced the data analyzed below, to provide a collection of typical cell shapes here. Right: trajectories as those shown here, make up the raw data that were analyzed statistically to find a suitable stochastic model for the motility of these keratinocytes. The black bar is $200 \mu \mathrm{m}$ long.

Furthermore, before we checked the equation of motion, we could check whether the data were consistent with various assumptions of symmetry and invariance on which the equation of motion is based. We found that the cells behaved in a manner consistent with the assumptions that their surroundings were isotropic, homogenous, and constant in time. This allowed us to average data over all directions, places, and times. This in turn improved the statistics of our investigation of the equation of motion, which proceeded as follows [12].

\section{Tailor-made theory replaces "one theory fits all"}

The theory in Eq. (5) states that for a given velocity $\mathbf{v}$ the acceleration is a stochastic variable with expectation value proportional to $\mathbf{v}$,

$$
\left\langle\frac{d \mathbf{v}}{d t}\right\rangle_{\mathbf{v}}=-\mathbf{v} / P .
$$

Figure $3 \mathrm{AB}$ shows that this is also the case for our experimental data for HaCaT cells. 

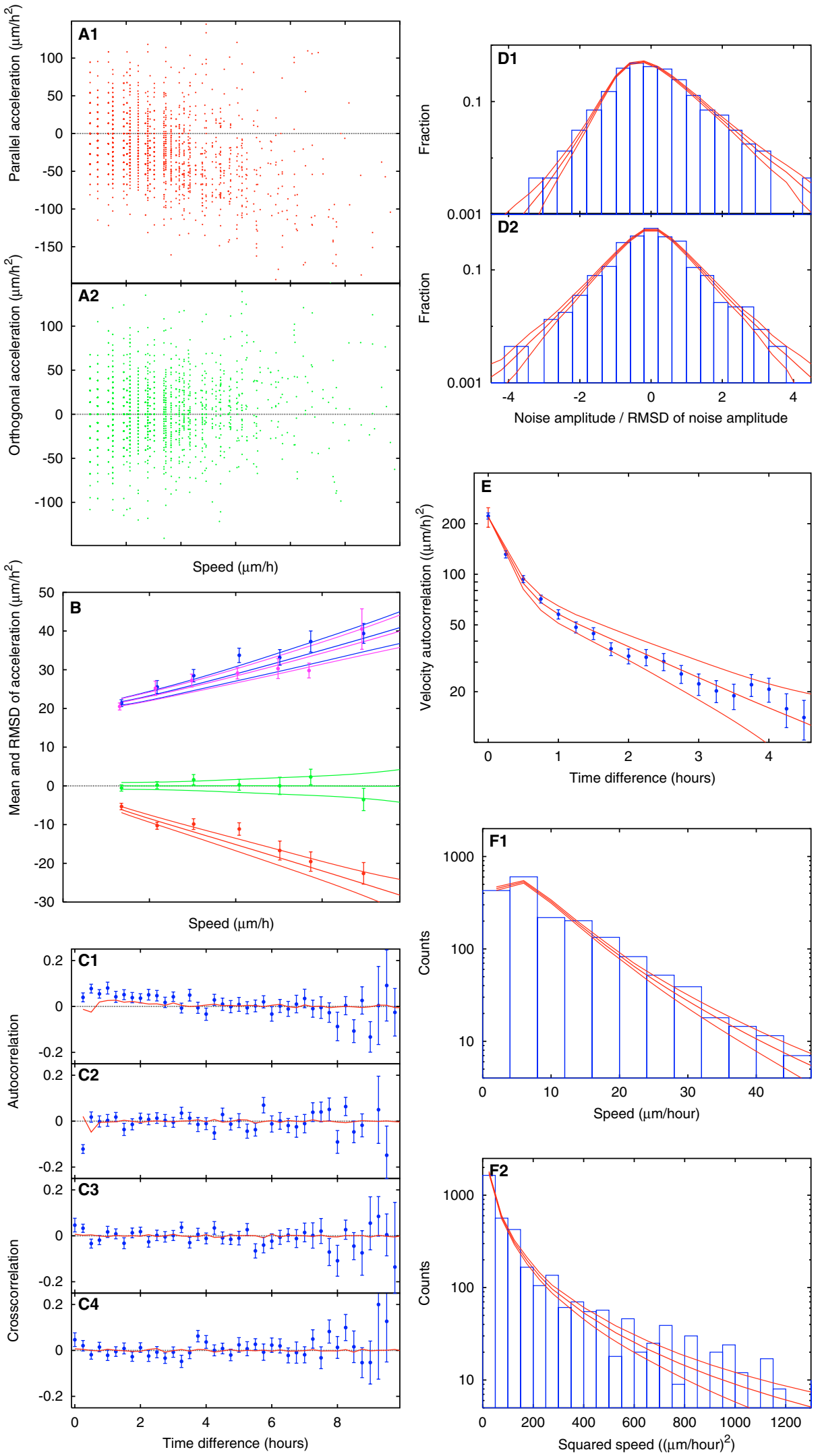
Fig. 3. Statistics accumulated from trajectories like those shown in Fig. 2. A) The two components of the acceleration, as functions of speed. Panels A1 and A2 show the acceleration parallel with, respectively orthogonal to, the velocity. These scatter plots show that the two functions contain random parts, like the acceleration in Eq. (5). B) Data points with error bars: Mean and standard deviation as function of speed for data shown in Panel A. Curves show the same quantities, plus/minus one standard deviation, calculated from the theory given in Eq. (9). C) Correlation functions for scatter shown in Panel A. Panels C1 and C2 show the auto-correlations of the two components, C3 and C4 show the cross-correlation between the two, for both signs of the time difference. The many values shown are almost all indistinguishable from zero. This suggests that the scatter in data can be modeled with uncorrelated noise, as in (4). This is an experimental result for the theory we seek. The curves shown are not fits to the data shown, but results of Eq. (9) after it has been fitted to data in Panels B, E, and F. D) Histograms of scatters shown in Panel A, measured relatively to the means shown in Panel B, and in units of the standard deviations shown in Panel B. The curves shown are not fits to the histograms shown, but results of Eq. (9) after it has been fitted to data in Panels B, E, and F. E) Velocity auto-correlation function, calculated from trajectories like those shown in Fig. 2. It is not a simple exponential as in (6). But a sum of two exponentials fit data perfectly. So we assume that the theory we seek has a velocity auto-correlation function that is a sum of two exponentials. The curves through the data points are that correlation function, plus/minus one standard deviation, computed with the theory in Eq. (9), after it has been fitted to the data shown here, and simultaneously to the data in Panels B and F. F) Histograms of speeds and (speed) ${ }^{2}$ read off trajectories like those in Fig. 2. The curves shown are the same speed distributions calculated from the theory in Eq. (9), after it has been fitted to the data.

The theory in Eq. (5) states also that

$$
\frac{d \mathbf{v}}{d t}-\left\langle\frac{d \mathbf{v}}{d t}\right\rangle_{\mathbf{v}}=\frac{d \mathbf{v}}{d t}+\mathbf{v} / P=(2 D)^{1 / 2} \eta
$$

where $\langle\cdots\rangle_{\mathbf{v}}$ denotes the expectation value for given velocity $\mathbf{v}$. The left-hand side in Eq. (8) can be estimated from recorded trajectories, and the right-hand-side shows that in the OU-process it is a white noise with the same speed-independent amplitude in both directions, parallel and orthogonal to the velocity.

Figure 3B, C, and D shows our experimental result for the left-hand side of Eq. (8). The data collapse in the upper part of Fig. 3B shows that the two components of this supposed noise have indistinguishable amplitudes in the two directions, as the noise term in Eq. (4) has. But the two amplitudes are clearly not independent of the speed, we see! They depend on the speed approximately like a first-degree polynomial. Here we see the experimental data reject the OU-process as a satisfactory model.

Figure $3 \mathrm{C}$ shows that on the time scale where it was measured, the two components of the left-hand side of Eq. (8) are uncorrelated with each other, and to a good approximation their auto-correlation is a delta-function, as the noise in Eq. (4) is. This approximation radically simplifies the mathematical task of constructing an alternative to the OU-process that reproduces experimental properties of HaCaT-cells' motility pattern.

Figure 3D shows that the distributions of experimentally measured values of the two components of the left-hand side of Eq. (8) also reject the OU-process as model. Clearly that they are not identical Gaussian distributions, as they are in the OU-process. Nor are they Gaussian at all.

The velocity auto-correlation function of the OU-process is a simple exponential, see Eq. (6). Figure 3E shows the experimentally measured velocity auto-correlation function. It is fitted perfectly by the sum of two exponentials, so again the experimental data reject the OU-process as model.

The data shown in Fig. 3 are so rich in information that-with a few assumptions favored by Occam's Razor-one can deduce from the data which theory it takes to describe the data, and this theory is unambiguously defined by the data [12]. Results from this theory are shown as the fully drawn curves passing through the data points in Fig. 3. It is given by the stochastic 
integro-differential equation

$$
\frac{d \mathbf{v}}{d t}(t)=-\beta \mathbf{v}(t)+\alpha^{2} \int_{-\infty}^{t} d t^{\prime} e^{-\gamma\left(t-t^{\prime}\right)} \mathbf{v}\left(t^{\prime}\right)+\sigma(v(t)) \eta(t),
$$

where

$$
\sigma(v)=\sigma_{0}+\sigma_{1} v,
$$

and Ito calculus is used, in accordance with the manner data are plotted in Fig. 3A. The integral over past velocities in Eq. (9) is called a memory-kernel. It shows that these cells have memory. This is no surprise: The polarity of the cytoskeleton of a moving cell is a manifest memory of direction, and while its instantaneous velocity depends on the activity of transient pseudopods, the fact that pseudopods are active depends on states of the cell the motion in a given direction lasts longer than the individual pseudopod, one would expect.

\section{Unrelated look-alikes}

Despite its more complex form, the HaCaT-model in Eq. (9) somewhat resembles the equally complex hydrodynamically correct theory for Brownian motion; see, e.g., [13, Eq. (1.6.15)]. The latter, not the OU-process, describes classical Brownian motion of a microscopic particle in a fluid with similar density. This might be seen as further co-evolution, with the HaCaTmodel being inspired by the hydrodynamically correct theory for Brownian motion. One of us did used the latter theory extensively $[14,15]$ just before constructing the former model. Shared environment, as opposed to origin, is sufficient to explain the similarity, however: the two theories respect the same space-time symmetries, both respect causality, and both theories are linear.

Linearity is simplicity, so wherever in modeling it is sufficient, one avoids going beyond it. This is why both models are linear. The theory for the motility of normal human dermal fibroblasts (NHDF-cells) also described in [12] is not linear, because the motility data inspiring it, do not permit it.

The Principle of Causality states that the future does not affect the present, including present rates of change of state variables. Only the past can do this. This principle is respected throughout physics, and we have of course built it into our motility model as well. This is why the rate of change of the velocity depends only on past and present velocities in both models. The integral kernels occurring in both equations are memory kernels in order to respect this principle.

In a homogenous, isotropic environment that is constant in time, there is no absolute position, direction, nor time. A theory for a dynamical system in such an environment consequently cannot depend on the position variable $\mathbf{x}$, nor can it depend explicitly on the time variable $t$, nor on explicit directions in space. The theory must be invariant under translations in space and time, and covariant with respect to rotation of direction, i.e., transform like the velocity vectors it describes. Because these space-time symmetries are shared by hydrodynamics and cells, neither theory depends on $\mathbf{x}$, nor explicitly on $t$, and both models transform like a vector under rotations. The latter transformation property does not ensure that the noise in the motility model is isotropic. The fact that it is here, is an additional and independent experimental result for HaCaT-cells. It may not survive a repetition of the experiment yielding data with better statistics. In the case of the NHDF-cells also studied in [12], there is no such isotropy of the noise.

\section{Fitting is not explaining}

The near-perfect agreement seen in Fig. 3 between the HaCaT-model in Eqs. $(9,10)$ and the experimental data for HaCaT-cells does not teach us much about HaCaT-cells, except that their motility can be modeled phenomenologically by Eqs. $(9,10)$. In [12], near-perfect agreement 
is found also between experimental NHDF-data and the NHDF-model inspired by those data. The success of this mathematical phenomenology raises the following questions:

1. Which aspects of the cells' locomotion are captured by the various features of the mathematical models? Specifically, what does the shortest of the two correlation times in the velocity auto-correlation function describe?

2. While a fit is no explanation, data missed by a fit demand an explanation. So what causes the transverse noise shown in Fig. 3C2 to be so significantly negative and different from the theory at time separation $15 \mathrm{~min}$ ? The equivalent data point for NHDF-cells at 15 min time separation is not seen in [12, Fig. 3]. This is a mistake due to a uniform choice of window on the second axis there. The data point falls below the window at -0.260 .02 , i.e., a highly significant $26 \%$ negative correlation.

3. The approach to phenomenological model building described above is generic in spirit. Does it work in other cases than the two studied in [12], or were we just lucky there?

4. Limited statistics certainly makes it easier to make a simple theory consistent with data. Does our approach to phenomenological model building survive improved statistics?

5. On the other hand, the space-time symmetries, which we relied on in model building, do not depend on statistics. Better statistics only makes it more demanding to realize those symmetries in the experimental setup. The NHDF-model contains the HaCaT-model as a special case, but is itself generic in form. So will better statistics merely result in a better experimental resolution of the $v$-dependence of $\beta(v), \sigma_{\|}(v)$, and $\sigma_{\perp}(v)$ ? Or will a more complicated memory kernel be necessary, or maybe even a non-linear dependence on past velocities?

The following sections present preliminary results that partially answer these questions.

\section{Dictyostelium discoideum}

The motile behavior of the cellular slime mould species Dictyostelium discoideum (strain AX4) was monitored in the absence of external signals. Vegetative Dictyostelium cells were placed on a pure agar surface right after harvest from a bacterial lawn. They were studied at low density, with more than 1000 cell diameters as the average intercellular distance, so there was no interaction between individual cells. Cell motions were observed under a $15 \times$ phase contrast microscope and recorded every 10 seconds for $8-10$ hours.

Figure 4 shows the same quantities as were plotted in Fig. 3, but for motile Dictyostelium discoideum. Their higher speed and better contrast makes it relatively easy to accumulate very rich data, as shown here. Comparing with Fig. 3, one sees that the error bars are so much smaller in Fig. 4 that the agreement between theory and experimental data is more striking, where it occurs. The $10 \mathrm{~s}$ time resolution in Fig. 4 is also much better than in Fig. 3, when measured relatively to the shortest correlation time in the velocity. This is seen as a larger number of data points falling on the short-lived part of the velocity auto-correlation function.

The two autocorrelation functions for the fluctuations in the acceleration about its mean value, Panel $\mathrm{C}$, show a negative correlation at short time separations, and a positive correlation at yet shorter separation is resolved in the orthogonal fluctuations in Panel C2. These correlations are already contained in the theory, as shown by the red curve. To understand this, one should note that with a memory kernel in the theory, as here, fluctuations in the acceleration at given velocity are not exactly equal to the white noise driving the model, but are partly explained by fluctuations in the memory term about its mean value [16].

In Panel D, the experimental values fall above the theoretical values for fluctuations larger than 3 RMSDs. Yet RMSDs of theory and experimental data agree with each other in Panel B. This apparent logical conflict is explained by the logarithmic second axis in Panel D: The discrepancies between the experimental and theoretical distributions occur where both distributions almost vanish, hence barely affect their respective RMSDs. Comparing with Fig. 3's Panel D, we see no similar discrepancy there, but also poor statistics where it would occur. 

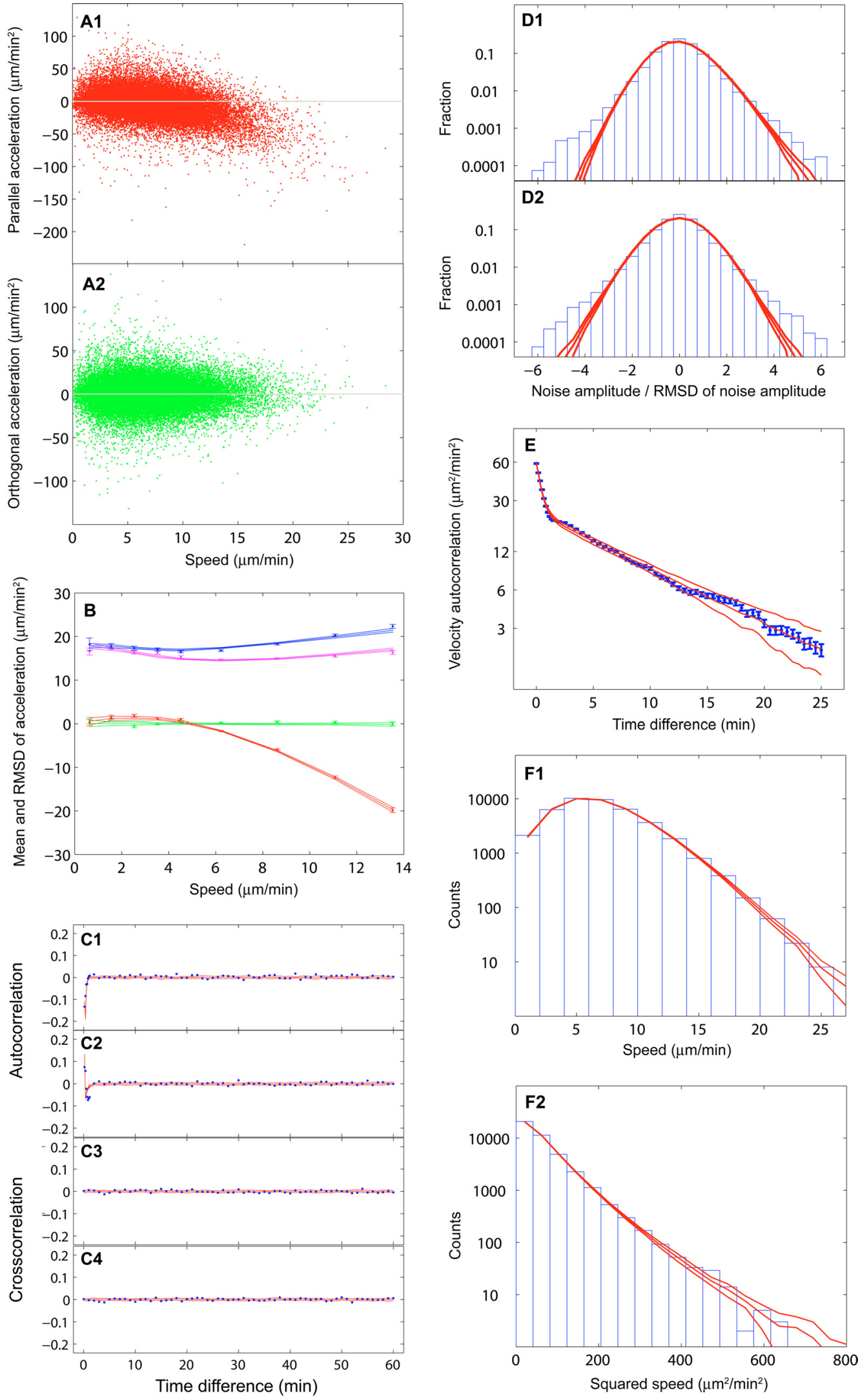
Fig. 4. Same as Fig. 3, but for richer data, recorded for motile Dictyostelium discoideum (strain AX4) in an isotropic, homogenous, and time invariant environment. The theoretical curves resulted from fits of what is essentially the NHDF-model presented in [12], except $\sigma(v)=\left(\beta_{0}+\beta_{2} v^{2}\right) /\left(1+\beta_{1} v\right)$, and similar expressions with different parameters for $\sigma_{\|}(v)$, and $\sigma_{\perp}(v)$. We found that these rational functions work better than second degree polynomials. (The Fokker-Planck equation that corresponds to the Langevin equation, which was solved and fitted here, must have a normalizable time-independent solution for the speed-distribution for the model to make sense. This results in conditions on $\beta(v)$ and $\sigma_{\|}(v)$ at $v \rightarrow \infty$, which are more easily satisfied by rational functions [16].)

As yet another effect of the better statistics in Fig. 4, one sees that at the "knee" of the velocity auto-correlation function, at time separations near $1 \mathrm{~min}$, where its short-lived component disappears, the theory does not quite follow the data. The data have a more complicated time-dependence at this "knee" than the model has. This complicated time-dependence presumably characterizes the "gait" of these slime molds, see below. For now, we notice that the NHDF-like model used here is unable to capture these finer details in the data. Similarly, there is no good agreement between the experimental noise distributions and the theoretical ones for amplitudes larger than three standard deviations. We may conclude that an NHDF-like model describes Dictyostelium data well to a degree, and beyond that degree a more elaborate model is necessary in order to capture all significant details in the data. To this end, it may be helpful to understand the dynamical origin of the various features in the motility data that we try to model mathematically. An attempt at this is given in the remainder of this review.

\section{Motivation for more modeling}

Above, we described some models for cell migration that resulted from a careful analysis of experimental data. Those were phenomenological models, and did not arise from a dynamical theory of cell migration. We therefore asked ourselves if it is possible to write down a simple, dynamic model for the cell migration that captures in a qualitative manner the experimentally observed behavior. Specifically, we wanted to construct a model that could reproduce the two time-scales observed in the experimental velocity autocorrelation function-see Figs. 3 and 4-as well as the curiously negative autocorrelation for the parallel and orthogonal components of the acceleration.

Recently it was pointed out that in two dimensions such an autocorrelation function can arise if the direction and the speed of a migrating cell fluctuate independently of each other [17]. This result was found by studying steady state probability density functions for the speed and the direction. Thus, the dynamics of the underlying stochastic process was not specified.

Quite generally, if in the stationary state the speed $v(t)$ and the direction of motion $\theta(t)$ vary independently of each other, and their variations can be characterized by rate constants $\beta$ and $\kappa$ respectively, then it follows rather trivially that the velocity autocorrelation function is

$$
\begin{aligned}
\langle\mathbf{v}(t) \mathbf{v}(0)\rangle & =\langle v(t) v(0)\rangle \cos [\theta(t)-\theta(0)]\rangle \\
& =\langle v(t) v(0)\rangle\langle\cos [\theta(t)-\theta(0)]\rangle \\
& =\left\langle v^{2}\right\rangle t e^{-\kappa t}+\left(\left\langle v^{2}\right\rangle-\langle v\rangle^{2}\right) e^{-(\beta+\kappa) t} .
\end{aligned}
$$

From this expression we see that there will be a fast decay with a rate constant of $\beta+\kappa$, followed by a slower one with rate constant $\kappa$. We also see that the fluctuations in speed and direction must be balanced rather precariously in order for two time-scales to be visible in an experimentally determined autocorrelation function: If $\kappa \gg \beta$-i.e., if the direction fluctuates much faster than the speed-only the single time-scale $1 / \kappa$ will be resolved. Furthermore, if the amplitude of speed fluctuations is small, $\left\langle v^{2}\right\rangle \simeq\langle v\rangle^{2}$, again only a single time-scale is visible, and again this time is set by $1 / \kappa$.

In the above analysis we made only three assumptions: (i) Fluctuations in speed and direction are independent of each other, (ii) the correlation times of these fluctuations are $\beta^{-1}$ and $\kappa^{-1}$, respectively, and (iii) the distributions from which $v$ and $\theta$ are drawn have finite variances. 
Thus, the above result is derived under slightly more general conditions than in [17], though still in the stationary state, and still in two dimensions.

In the following, we are going to relax the requirement of independence of speed and direction. What we gain in so doing is generality and, we will argue, a higher degree of realism. The price we pay is the loss of analytical tractability. Thus, we turn to computer simulations.

\section{Cell motility as a stick-slip process}

Cells move by protruding their front and retracting their rear $[18,19]$. As a front protrusion sticks fast to the substratum, internal contractile forces build up. Eventually, the adhesion at the rear (or occasionally the front) breaks down, and the cell slips over the surface, until the forces are balanced anew. Thus, cell migration can be thought of as a stick-slip process in which random-duration events of sticking and slipping alternate to produce a, more or less, irregular mode of motion.

To model this process, we start out with a simple one-dimensional model and then add to it layers of complexity, and move to two dimensions, as we refine the model to reproduce the output of experiments with real cells.

\subsection{Cell motility as a stick-slip process-simplest version}

Consider a point that is confined to move in one dimension. At any given time, the speed of the point is either zero or a positive constant. The direction of motion is either to the left or to the right. For the sake of illustration, let the point start out with a positive speed, moving to the right for a duration of time that is drawn from an exponential distribution with timeconstant $\tau_{1}$. The point then pauses for a duration of time that is also drawn from an exponential distribution, but with a different time-constant $\tau_{0}$. That is, both moves and pauses are given by Poisson processes. ${ }^{2}$ After the pause there is a small, but finite, probability of the point moving in the opposite direction: Think of the point as having a vector $\mathbf{p}$ associated with it, which always points in the direction of motion; when the point pauses, it still has an orientation of coming motion, but during the pause this orientation may change direction (left/right) with some probability $c$ per unit time. Thus the direction of the vector denoting orientation may change in each pause, and change more than once, but it never changes during a move.

This fictive vector $\mathbf{p}$ is meant to represent the polar structure of a cell. In only allowing directional changes when the point is not moving, we model the fact that cells are polar when moving, but may lose or change their polarity when not moving. This change in polarity could, e.g., be due to a reorganization of cytoskeletal organizing molecules in the leading edge of the membrane, with a consequent change in the direction of growth of the next pseudopodium, and thus of the direction of motion.

In this model, the fluctuations in direction are not independent of the speed. Only when $\tau_{0} \gg \tau_{1}$ do we expect agreement with the analytical result for the velocity autocorrelation function given in Eq. (11)

The various models described above and below were examined by creating time-series of positions with a fine temporal resolution $\delta t$, and then sampling these series with a much coarser time-resolution of $\Delta t$. Measured velocities $v_{i}=\left(x_{i}-x_{i-1}\right) / \Delta t$ and accelerations $a_{i}=\left(v_{i+1}-\right.$ $\left.v_{i}\right) / \Delta t$ are defined as in $[12]$.

The result of a simulation of this one-dimensional stick-slip model is shown in Fig. 5. Panel E shows the desired velocity autocorrelation function with two time-scales. For the choice of parameters indicated in the caption, it is fitted perfectly by the sum of two exponential functions. However, Panels A and F1 show that we are still some way from a realistic model of migration.

\footnotetext{
${ }^{2}$ We might refer to this shuffling manner of stochastic motion as The Poisson Shuffle, as long as it is not confused with the mathematics of shuffling of playing cards.
} 
A

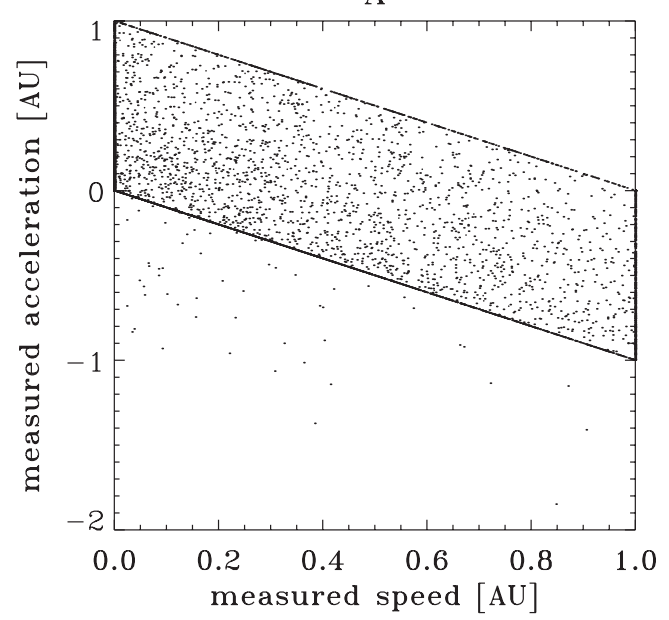

$\mathrm{B}$

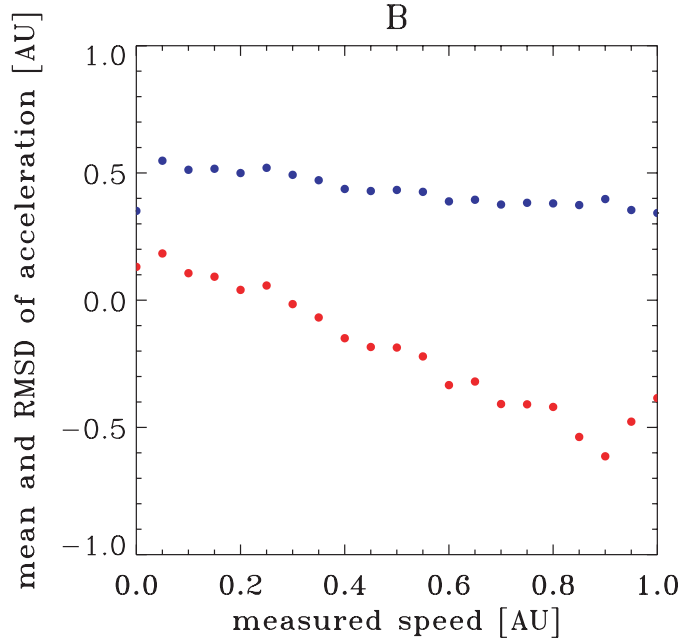

$\mathrm{E}$

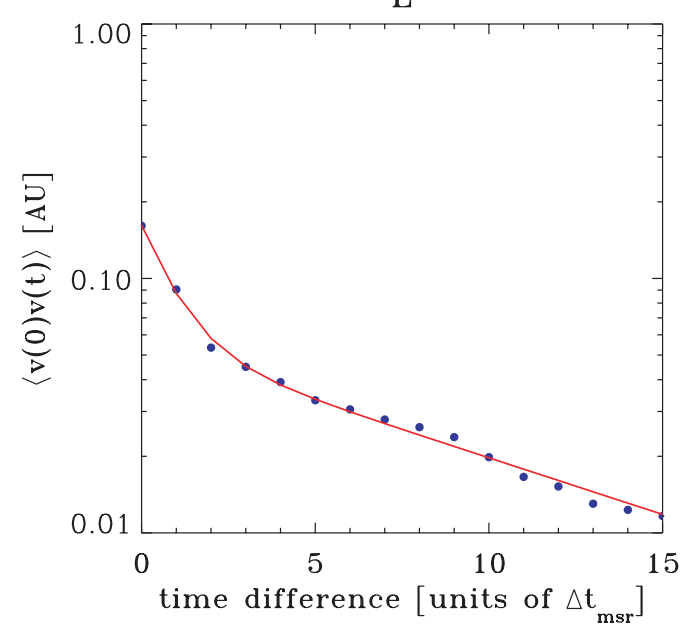

F1

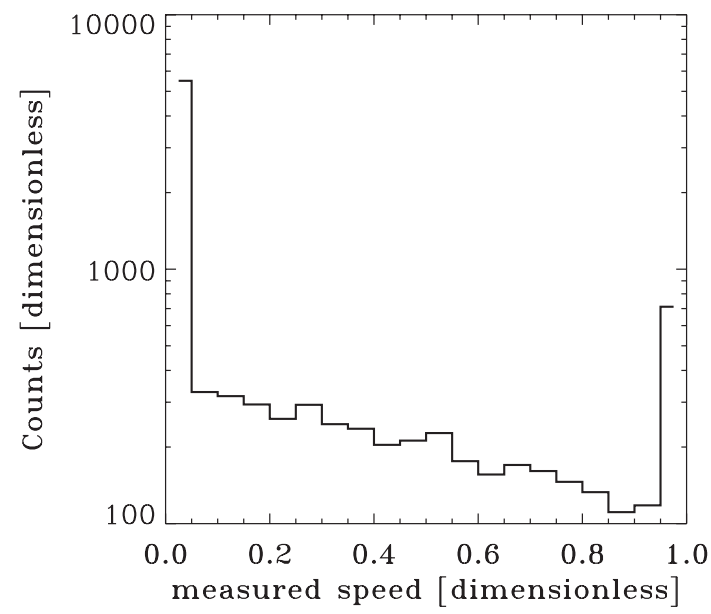

Fig. 5. Simulation of the one-dimensional stick-slip process. (A) The measured parallel acceleration as a function of the measured speed. (B) The mean (red) and RMSD (blue) of the measured parallel acceleration as a function of the measured speed. (E) The velocity autocorrelation function. The red line is a fit to the data of the sum of two exponential functions with characteristic times $t_{1}=1.0 \Delta t$ and $t_{2}=15.2 \Delta t$. (F) The measured speed distribution. Parameters used in the simulation $\tau_{0}=2.50 \Delta t$, $\tau_{1}=0.75 \Delta t$ and $c=0.07 \Delta t^{-1}$. The speed of the point is either zero or one.

\subsection{Stick-slip process with exponential speed distribution}

A simple improvement of the above model is achieved by allowing the speed in the moving state to be drawn from, e.g., an exponential distribution. Among other effects, this leads to a linear dependence of the root-mean-square-deviation (RMSD) of the parallel component of the acceleration on the speed, as is observed in experimental data [12].

\subsection{Stick-slip process with multiple pseudopodia}

Staying in one dimension, further sophistication is brought about by letting the speed of the point be determined by the sum of several processes, each one identical to the Poisson process just described: Each process can be thought of as an individual pseudopodium, which is active 

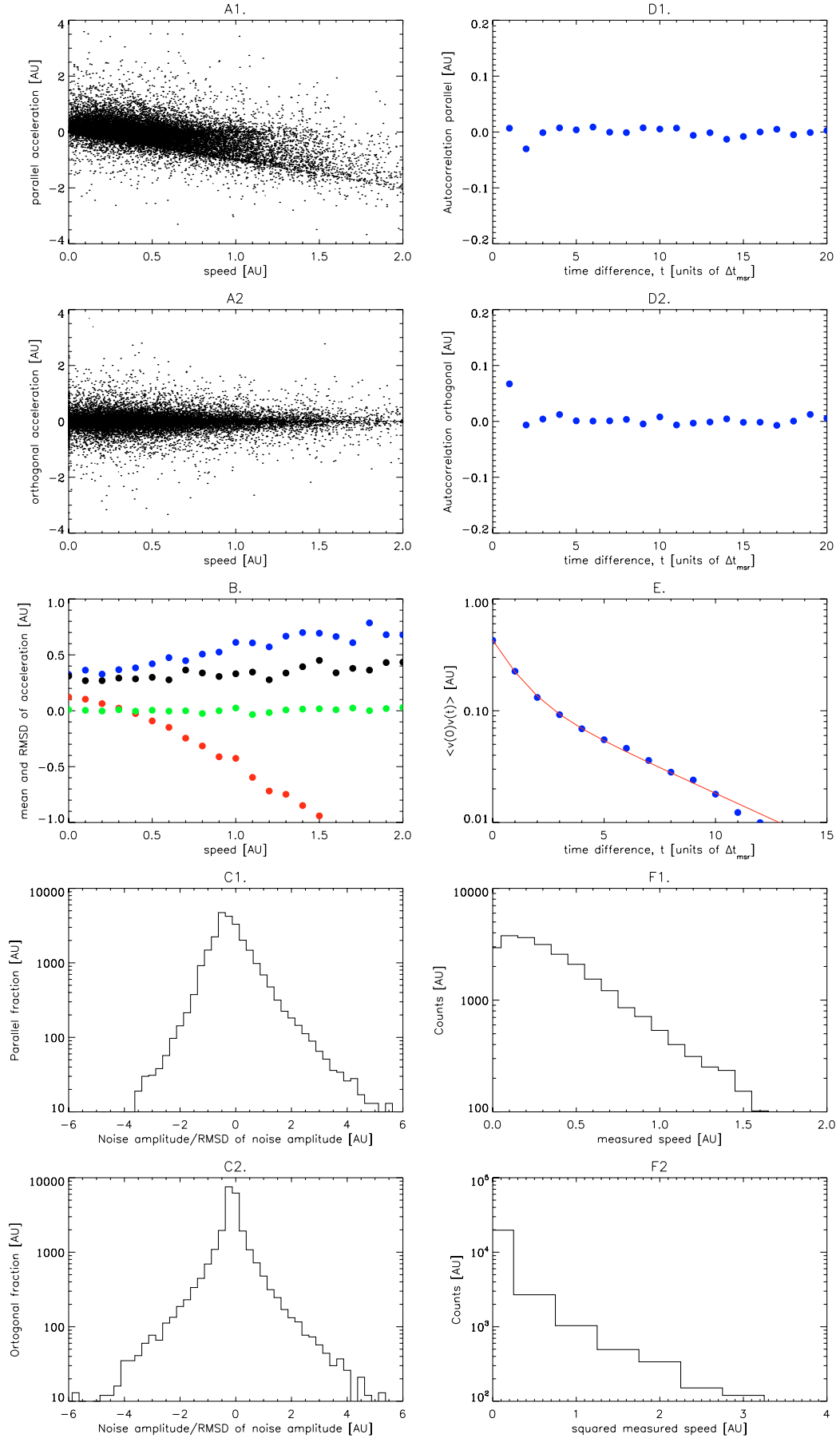

Fig. 6. (A1/A2) The parallel/orthogonal accelerations as functions of speed. (B) Mean parallel (red) and mean orthogonal (green) acceleration as functions of speed and RMSD of the parallel (blue) and orthogonal (black) accelerations as functions of speed. (C1/C2) Noise amplitudes of the orthogonal/ parallel accelerations in units of RMSD. (D1/D2) Autocorrelation functions of the parallel/orthogonal acceleration's deviations from its mean value. (E) The velocity autocorrelation function. The red line is a fit of two exponentials with characteristic times $t_{1}=1.1 \Delta t$ and $t_{2}=4.8 \Delta t$. (F1) The speed distribution. (F2) The squared speed distribution. 
for a while, during which it adds to the speed of the cell, and after which does not for a while. The point thus exist in a pausing state when none of the pseudopodia are active, and in a moving state when at least one pseudopodium is active. The speed is given by the sum of one or more exponentially distributed stochastic variables, i.e., the speed is drawn from a gammadistribution and auto-correlated in time beyond the active period of individual pseudopodia. We fix the maximum number of pseudopodia to $n$; the number of active pseudopodia is then somewhere between zero and $n$ at any given time.

\subsection{Stick-slip process in two dimensions}

The final refinement of our model consists in allowing the direction of motion to vary smoothly between $\theta=0$ and $2 \pi$. This is obtained by vector addition of velocities, corresponding to the action of pseudopodia in differing directions. In two dimensions we can define two directions: Parallel and orthogonal to the instantaneously measured velocity. A plot of the autocorrelation function for the parallel and orthogonal components of the acceleration is shown in Fig. 6, Panels D1 and D2. For zero time-separation these functions equal one by normalization. The values found for non-zero time-separation seem to vanish except, possibly, for a negative auto-correlation at separation $2 \Delta t$ in Panel D1, and a more convincing positive correlation at separation $\Delta t$ in Panel D2.

Comparing with the experimental values in Fig. 3, Panels C1 and C2, we see similar differences to a pure delta-function correlation at small time-separation, but there it is the transverse fluctuations that are anti-correlated, and the longitudinal fluctuations are slightly positively correlated. Figure 4, Panels $\mathrm{C} 1$ and $\mathrm{C} 2$, on the other hand, agree with our theoretical result, for what the latter is worth. For now, we can only say that if we are optimistic, it seems that this kind of simple model may be able to reproduce correlations similar to those seen in the experimental data, but more work is needed before we can conclude on this point.

\section{Conclusion}

We have formulated a hierarchy of increasingly complex geometrical models for cell migration, which has the following properties in common with the experimental motility data for NHDFcells:

- The amplitude of the parallel component of the acceleration, $a_{\|}$, depends on the cell's speed as a first-degree polynomial.

- The amplitude of the orthogonal component of the acceleration, $a_{\perp}$, is almost independent of speed, but decreases a little, in a manner that can be described with a first degree polynomial.

- The velocity autocorrelation function is given by the sum of two exponential functions.

- The autocorrelation functions for $a_{\|}$and $a_{\perp}$ are approximately delta-functions, the approximation being limited by small non-zero values at small time-lags.

We conclude that with the rich data that one now can record and process, one should not be satisfied with the simplest possible model for persistent random motion, the OU-process. "One size fits all" is no longer true, if it ever was. Motility models can be made to measure. Here we have only presented the first phenomenological steps of that process: How to plot and read motility data in a manner that reveals mathematical properties of the theory sought. That done, it is another task to construct a model with the properties demanded. If that can be done, it is yet another task to decide whether the theory is unique or not. Two examples of such theories and their derivation are given in [12].

D.S. was supported by the Danish Research Agency's Graduate School of Biophysics and Tamas Vicsek. L.L. and E.E.C. were supported by grants from the NIH and the Boroughs-Welcome Fund. S.M. was supported by the Graduate School of Biophysics. N.B.L. was supported by the Danish Technical Research Council. HF thanks the organizers of the workshop Active Motion and Swarming, Berlin, December 4-6, 2006, for an inspiring meeting. 


\section{References}

1. A. Pais, Subtle Is the Lord: The Science and the Life of Albert Einstein (Oxford University Press, 2005)

2. J. Ingen-Housz, Verm. Schriften physisch-medicinischen Inhalts, 1784, available at www .physik. uni-augsburg.de/theo1/hanggi/History/BM-History.html

3. J. Ingen-Housz, Paris, 1789, available at www . physik. uni-augsburg.de/theo1/hanggi/History/BM-History.html

4. A. Einstein, Investigations on the Theory of the Brownian movement, edited by R. Fürth, translated by A.D. Cowper (Dover Publications, Inc., 1956)

5. K. Przibram, Pflgers Arch. Physiol. 153, 401 (1913)

6. R. Fürth, Ann. Phys. 53, 177 (1917)

7. R. Fürth, Z. Physik 2, 244 (1920)

8. L.S. Ornstein, Proc. Amst. 21, 96 (1918)

9. G.E. Uhlenbeck, L.S. Ornstein, Phys. Rev. 36, 823 (1930)

10. P. Langevin, C. R. Acad. Sci. (Paris) 146, 530 (1908), translated and commented in [20]

11. M.H. Gail, C.W. Boone, Biophys. J. 10, 980 (1970)

12. D. Selmeczi, S. Mosler, P.H. Hagedorn, N.B. Larsen, H. Flyvbjerg, Biophys. J. 89, 912 (2005)

13. R. Kubo, M. Toda, N. Hashitsume, Statistical Physics, Vol. 2 (Springer-Verlag, Heidelberg, 1985)

14. K. Berg-Sørensen, H. Flyvbjerg, Rev. Sci. Instrum. 75, 594 (2004)

15. K. Berg-Sørensen, E.J. Peterman, T. Weber, C.F. Schmidt, H. Flyvbjerg, Rev. Sci. Instrum. 77, $063106(2006)$

16. L. Li, H. Flyvbjerg, E.C. Cox (2007) (in preparation)

17. F. Peruani, L.G. Morelli, Phys. Rev. Lett. 99, 010602 (2007)

18. D.A. Lauffenburger, A.F. Horwitz, Cell 84, 359 (1996)

19. S.M. Rafelski, J.A. Theriot, Annu. Rev. Biochem. 73, 209 (2004)

20. D.S. Lemons, A. Gythiel, Am. J. Phys. 65, 1079 (1997) 